\title{
Saudi University Girl's use of Social Networking "WhatsApp" and its effect on Gratification
}

\section{Dr. Aliaa Abdel Fattah Ramadan}

\author{
Assistant Professor and Head of the Educational Media Department - Tanta University \\ Email: aliaaramadan11@gmail.com
}

Received: 29 Nov. 2019 Revised: 03 Dec. 2019 Accepted: 23 Dec. 2019 Published: 15 January 2020

\begin{abstract}
WhatsApp as a social network application is widely used worldwide due to Wi-Fi connections on mobile phones. This current study aims at tracking the reasons and motivation of its use among Saudi University girls (200 students) via designing a questionnaire. The students 'responses were coded, and SPSS programs were used to analyze the responses of the sample. The results represent the frequency of the reasons behind using WhatsApp.
\end{abstract}

Keywords: Social Network - WhatsApp - Gratification.

\section{Introduction}

The world has witnessed a lot of global developments, the cognitive explosion, the popularity of satellite TV, Satellites, Internet, Mobiles phones and communications, which have brought changes to different fields of life.

In this regard, (Al-Qaoud, 2006) emphasized that the Internet serves the social sphere because it provides text and audio-visual areas of communication.

Internet services, as a form of technological development, are diverse and they are accessible to all at all times everywhere. The internet has entered mobile phones after computers, the third generation of communications. Through Wi-Fi, the connection is cheaper. WhatsApp as a mob application is an example of social communication via the internet. It is considered by others to be an instant messaging application for exchanging messages among phone users.

The Center for American Strategic Studies has indicated that the use of communication sites in Saudi Arabia comes fourth among the people of the Cosmic village and that the use of WhatsApp has been at the forefront of such means (AlKhalaf, Amasha, and Reeves, 2019) 
The study aims at tracking the use of the WhatsApp application, as its availability via Wi-Fi internet connection on smartphones encouraged Saudi girls University students to use it anywhere as long as it is available all the time. It also aims at studying its effect. The problem of the study: Social networking is one of the most notable innovations on the Internet that has changed the culture and the mindset. Millions of people around the world have been able to exchange activities, disseminate their information and interact with each other through the use of social networks and new media like Facebook, Twitter, and finally the "WhatsApp" program.

Because of the widespread use of smartphone technology among Saudi university girls, especially the "WhatsApp" service, where it has become noted that a university girl spends a long period of the day dealing with the phone to use this service, even during lectures, and even when she is with her colleagues, who looked forward to the need to consider that interest and the widespread use of this service by girls to determine the benefits of their use of the program.

\section{Methodology:}

\section{1) Type of the study:}

This study is one of the descriptive studies that aim at tracking a particular phenomenon to describe, analyze, and identify the reasons behind it namely the use of WhatsApp among Saudi University girls in relation to the previous studies. The survey, which is considered an organized effort that enables the tracking of a phenomenon to characterize it, does not stop at the mere description of it but tries to touch its objective reasons to achieve the objectives of the study and answer its questions.

\section{2) Study Population:}

The researcher chose Saudi university girls to be a research sample

\section{Study Sample:}

A random sample of 200 Saudi girls was withdrawn from two universities: Taiba University in Madinah, Saudi Arabia.

\section{Data Collection Method:}

A questionnaire was designed to include measurable study variables.

\section{Data Analysis Method:}

After the data was reviewed, all the forms were manually reviewed. The data was coded and entered into the computer. SPSS was used to process the data statistically to extract the frequencies and percentages. The Pearson correlation coefficient was also used to study the intensity of the correlation. The differences between the theoretical and practical specialties, as well as the test: "One Way Anova" to compare the responses of the members of the study according to the intensity of use. The results of the study were explained considering the previous studies and the proper scientific inputs for the study.

Results of the study: To answer the first question: What are the motives and reasons for the use of social networks?

\section{What are the reasons for your interaction with social networks?}


Table (1)

\begin{tabular}{|c|l|}
\hline Percentage $\%$ & \multicolumn{1}{|c|}{ Reasons for using social networks $\%$} \\
\hline $97 \%$ & $\begin{array}{l}\text { Enable communication, chat with friends, and follow } \\
\text { up on the latest developments }\end{array}$ \\
\hline $93 \%$ & Expressing opinion and presenting issues and ideas \\
\hline $88 \%$ & Social interaction with others via communication \\
\hline $71 \%$ & Desire to obtain information and gain experience \\
\hline $68 \%$ & Discussions in the field of study \\
\hline
\end{tabular}

Table 1 shows that the most important reason why girls interact with social networks is to make contact with friends and follow up the latest developments by $97 \%$, followed by expressing opinion and putting issues and ideas at $93 \%$, followed by interaction social communication with others through the means of communication by $88 \%$, followed by the desire to obtain information and gain experience by $71 \%$, and then exchange dialogue and discussions in the field of study by $68 \%$.

The researcher explains that: social networks achieve a good virtual environment, in addition to the ease and cheap cost of this communication medium, and also has higher advantages than other types of communication, as they can communicate with voice and pictures if they wish to, which also confirms That the public of social networks is very active and has specific goals and objectives that seek to achieve and satisfy them from this use.

The results of this study agree with the findings of (Suzant, Kinney, 2012), which found that the new media - social networking - have made content richer through instant signals, feedback, and feedback time. New media has slowed down decision-making on certain topics, as a result of the many and varied views on these issues.

The results of this study also agreed with the study (Sacide Guzin Mezman, 2009), which found that the most important reasons for the use of social networks is the ease of use, social factors supporting social networking, a social networking environment, and a permanent environment for innovation and creativity.

The results of this study supported the results of the (Uwem Akpan, 2013) study. The study found that young people in Oyo, Nigeria, use at least 46 social networking sites, indicating that their interaction with the Internet taught them intelligence in dealing with global social networking sites. The study found that young users of social networking sites are very active in dealing with these networks and use these networks to satisfy specific purposes and objectives.

\section{What are your motives for using social networks?}

Table (2)

\begin{tabular}{|l|l|}
\hline$\%$ & Motives for use of social networks \\
\hline $64 \%$ & Saturation in need for knowledge \\
\hline $64 \%$ & The latest news in the world \\
\hline $44 \%$ & Diaries \\
\hline $75 \%$ & Upload photo albums, music, or video files \\
\hline $32 \%$ & Spending leisure time \\
\hline
\end{tabular}




\begin{tabular}{|l|l|}
\hline$\%$ & Motives for use of social networks \\
\hline $46 \%$ & Habit \\
\hline $34 \%$ & Entertainment \\
\hline $65 \%$ & Active for me \\
\hline $95 \%$ & Social interaction and communication \\
\hline $82 \%$ & Send messages \\
\hline $63 \%$ & Comment on friends' message \\
\hline $92 \%$ & Communicate with groups of common interest \\
\hline $76 \%$ & Gain new skills and experience \\
\hline
\end{tabular}

Table 2 shows that the most important motivation for female students' use of social networks is "social interaction and communication with others" at $95 \%$, followed by communication with groups of mutual interest at $92 \%$, followed by sending messages to friends and associates at $82 \%$, followed by saturation in need of knowledge, access to new news in the world by $64 \%$, and so ended with the phrase habit by $46 \%$, entertainment by $34 \%$, and leisure time by $32 \%$.

The findings of this study are in line with the results of the study (Al-Mansour, 2012), which found that social networks are considered one of the largest and most widely distributed web sites to provide communication between individuals and groups that enable them to communicate and exchange ideas, opinions, information, and videos.

Sites have proliferated in recent years and become the largest sites in the webspace and are still continuing in the rapid horizontal spread, which are providing communication service among its members, where a user can connect to a friend through the site and allows the user to control the content that appears on his or her page, showing only what friends add to their writing, photos, and clips. "The most popular sites are Facebook and Twitter."

Commenting on the results of the study, Peter Cameron, General Manager of IFG, said: "This is an exciting idea. Most children in today's world have a digital presence as soon as they reach two years of age. Later, adding to the need to know the privacy settings they follow on their personal pages, otherwise you will find that everyone can access your child's photos rather than just friends and family, the Canadian newspaper Montreal Gazette reported.

\section{What are the positives of social networks?}

\section{Chart No. 3}

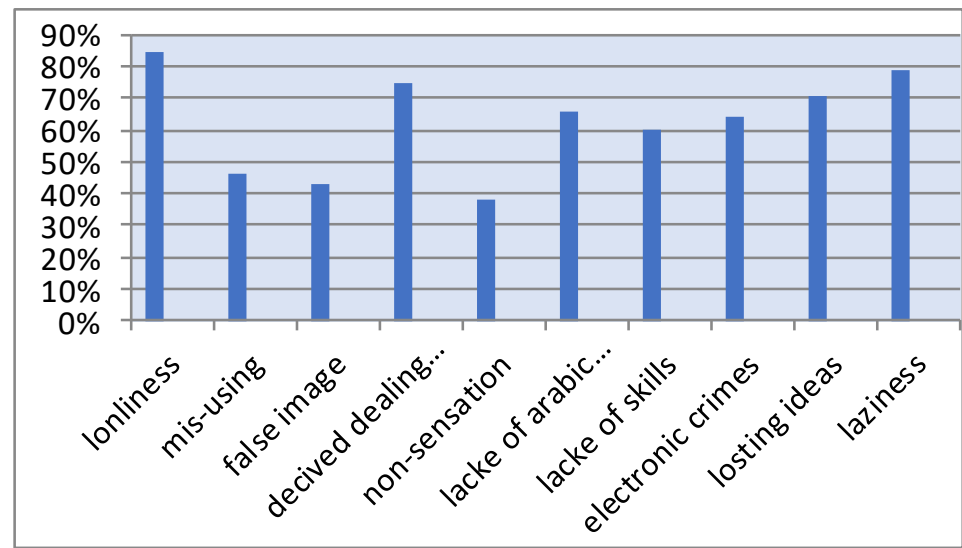

Figure (3) shows that the most important positive aspects of the social networks from 
the point of view of the sample is "easy, and smooth communication" by $91 \%$.

The researcher explains that: social networks have brought people closer and facilitated communication with people from the ends of the earth and made the world look like a small village in terms of ease of communication and exchange of information and experiences.

This result confirmed the further result of deepening the concept of participation and effective communication with others by $87 \%$, followed by "the interaction between the sender of the information and its reciever" by $83 \%$ and the speed of the transfer of the news and strengthen it with live images expressed by $82 \%$, followed by the development of skills in the use of computers and networks by the user by $74 \%$, followed by opened horizons of communication between individuals, peoples and organizations in an unprecedented manner by $72 \%$, followed by "exchange of experiences, cultures, data, opinions and ideas in transparency and freedom" by $68 \%$, followed by "able to contribute significantly to achieving the development objectives of its potential and characteristics by $52 \%$, and finally focused on the total discoveries and technical additions that have led to net unprecedented results and benefits for humanity "by $41 \%$.

The results of this study also agreed with the results of the study (Kateb, 2012), which proved that the social networking networks as a means to share experiences and that it has the potential and characteristics that can contribute significantly to achieve the goals of administrative development significantly One of the most important characteristics is the participatory and interactive capacities of these networks And interactive networks that make it possible to share and inform people about management development programs and also to allow them to interact directly with them, they also lined with the results of the (Goff, 2009) study, which found that social networks are more positive than negative. Studies such as the Digital Youth Project and the recently published "Child Safety and Technology on the Internet" report by Harvard University show that the Berkman Center for Internet and Society That social networks have gained a special place in American culture cannot be reversed.

\section{What are the disadvantages of social networks?}

\section{Diagram No. 4}

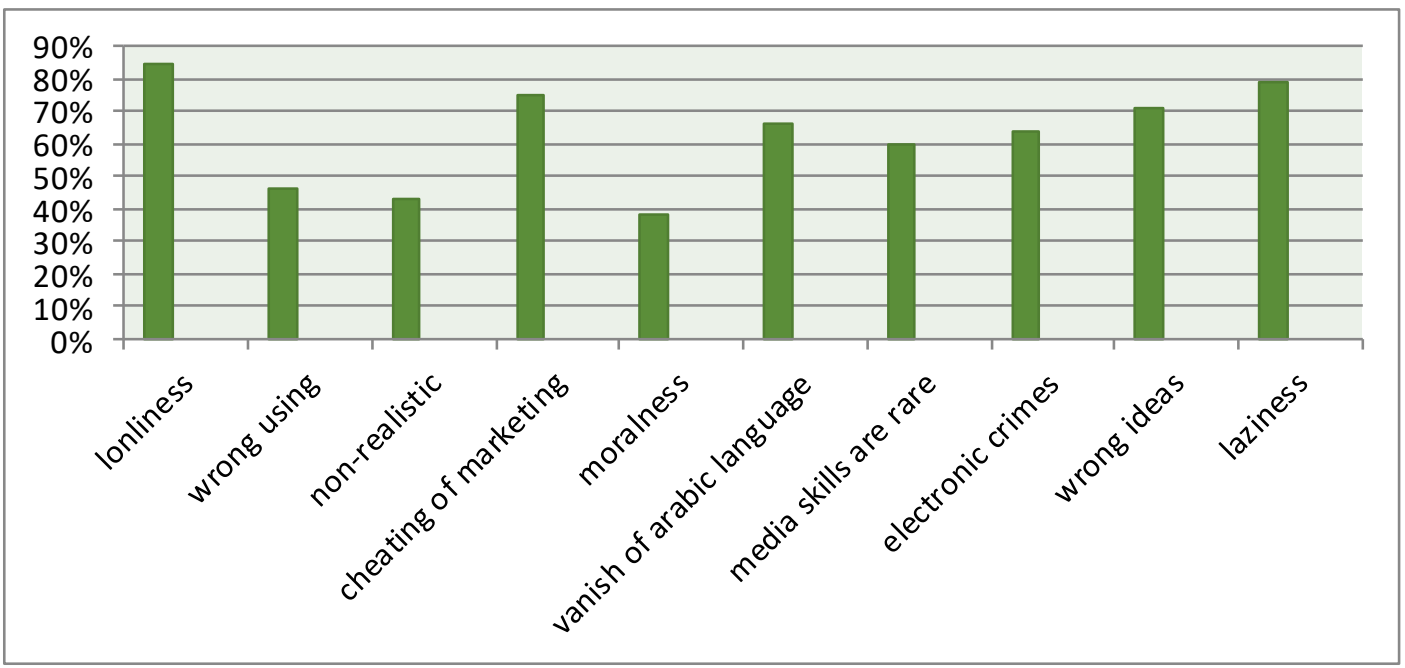

The "4" plan indicates that the most important disadvantages of social networks are that 
they "achieved social isolation" by $85 \%$. The researcher explains that: Social networks are the most important disadvantages of wasting a very large time as a result of spending a great time in communication with others, which leads some of the social isolation satisfied with virtual communication instead of personal communication. It is a means of technology that helps to weaken the social relations between members of the family; it is the disadvantages of social networks social isolation, resulting from the excessive use of young people and the result of the excessive use of young people, which makes the young man isolated from the social environment; so that he may know only the friends and friends imaginary in the chat room; this isolation has a negative impact on young people, including: concern about study and repetition, and the weak social interaction between him and his family members.

This result confirmed the other result that "it leads to laziness, and preoccupation with reading" by $79 \%$. Followed by "phishing" by $75 \%$, followed by "language vanishing resulting from the emergence of a social dialogue language" of $66 \%$ followed by "exposure to stereotypes such as violence, negative security and gender" by $71 \%$, followed by "exposure" For the crimes of electronic crimes such as: the crimes of hacking sites and files and personal computers "by $64 \%$, followed by" weak media skills or lack of media and user of the network "by $60 \%$, followed by" may be used by some wrong form, which is not held accountable "by $46 \%$ It does not convey the true picture of social reality "by $43 \%$ "The non-moral sense is due to the huge amount of information filled with violence and destruction" is $38 \%$.

The results of this study were consistent with the results of (Khidr, 2009)study. The study found that there are bad psychological effects for the use of social networks, including the elimination of the correct social relations based on interpersonal interaction, where young people tend to social isolation and are not socially optimistic. The people who meet them in the real world and this is one of the most dangerous negative social effects of the site Facebook.

The above shows that the positives of social networks are more negative, and this shows that after years of social networking there were parents who say "I do not want any of these things to come to my house", then these parents realized that they had no choice, and now they themselves are teaching their children the technical skills to deal with these networks, because from their point of view they have become a necessity dictated. What is the rate of university girls' use of WhatsApp?

What are the most social networks you use?

Table (3)

\begin{tabular}{|c|c|}
\hline$\%$ & Most social networks used by \\
\hline$\% 99$ & Watts App \\
\hline$\% 82$ & Twitter \\
\hline$\% 78$ & Face book \\
\hline$\% 75$ & You tube \\
\hline$\% 57$ & Instagram \\
\hline$\% 35$ & Cake \\
\hline
\end{tabular}

The most popular social networks are "WhatsApp", 99\% followed by Twitter, 82\% followed by Face book, 78\% followed by YouTube, 75\% followed by Instagram, 57\%. Cake is $35 \%$.

The researcher explains this: How effective and important the use of social media, "WhatsApp" and Twitter, Face book and YouTube in the social life of girls, in 
particular "WhatsApp because the uniqueness of this program from other programs that in their view - the best and easiest to use, As a result of the excellence of its design and ease of use, and to collect all the pros and possibilities desired by the girl whatever their interests and specialization, as the researcher returns to the desire of the Saudi girl continues to keep up with everything that is new in the world of technology, especially social networks, his appearance with a high degree and then followed by the transformation use Twitter since the beginning of his appearance, and then the newly WhatsApp, due to the achieved by these means for the girl from the ease and effectiveness in communicating with others.

The results of this study agreed with Phil (Murphy, 2012), whose study attempted to answer the following question: Why do teens turn from Face book to WhatsApp? The result was a study of a sample of adolescents - where teens use WhatsApp on a large scale - that Facebook is a traditional means of communication and interaction just for advertising, but the WhatsApp has many advantages that satisfy their desires.

They also agreed with the results of the (Chirinda, 2012), which aimed to identify the reasons for the transformation of networks from Facebook to the WhatsApp, and found that many talk about the social networks and technology associated with them and adopted by users around the world, Facebook, Google, Twitter and other similar services or networks, now everyone is talking about WhatsApp as the most important IM applications, and as the most important multimedia messaging services, so these new devices and applications add or complement other means such as Facebook and Twitter, and with all these changes that $\mathrm{W}$ lightning speed, it was not very easy to understand exactly where we are going, the smart phones getting smarter and despite their small size, but it is very strong in the field of computing, the beginning was habitually Internet cafes, open sense.

\section{How long have you been subscribing to WhatsApp?}

Table (4)

\begin{tabular}{|c|c|}
\hline$\%$ & Time of using watts app \\
\hline$\% 82$ & 6 months \\
\hline$\% 18$ & from year \\
\hline
\end{tabular}

It is clear from the table that $82 \%$ of the sample used the WhatsApp six months ago and that $18 \%$ of the sample used it for one year.

The researcher explained that: the modernity of the service of WhatsApp, where it has seen a remarkable and increasing popularity in the recent period, which led to the spread and penetration, and the desire of girls to use, as by establishing a students in the university has expanded the circle of use and awareness of its benefits, which has led to an increase in popularity and spread quickly among girls, and is a kind of social networking applications such as Facebook, Twitter and other software that aims to communicate between people in an easy.

In addition, this service stands for a quantum leap and a vital breakthrough that has fundamentally changed the means of communication in general and mobile. Answer the second question.

\section{C) How often do you use Voices on social networks?}


Table (5)

\begin{tabular}{|c|c|}
\hline$\%$ & Usage Rate for Watts AP \\
\hline$\% 91$ & Always \\
\hline$\% 7$ & Sometimes \\
\hline $2 \%$ & Rarely \\
\hline
\end{tabular}

Table (5) shows that the percentage of those who regularly use social wats is $91 \%$ and sometimes use them at $7 \%$ and rarely $2 \%$.

The researcher explains that: It is an easy to use in the completion of communication between the sender and the future and its cost suitable for all segments of society, and compensates for the use of expensive SMS, and helps to exchange dialogue and discussions for work or study and lectures and exchange of experiences supported by images and availability at any time.

The results of this study are in line with the results of the (Lund, 2013)study, which concluded that the WhatsApp service is a high-quality service for sending messages, contacts, multimedia files, or even your own site from any platform once downloaded, installed and entered your phone number. Your contacts, regardless of the operating system they use, are on the one hand and on the other hand can work a simple, easy, and attractive interface, and the WhatsApp has many great features

You can create a list of names, create chat groups on a specific theme, add this to the available chat settings such as font size, font typesetting, your nickname and message status as well as notification settings such as ringtone identification and vibration.

The researcher believes that all these features available in WhatsApp have prompted the Saudi girl to use it permanently. Answer the third question.

\section{D) How many hours do you use wattage daily?}

Table (6)

\begin{tabular}{|c|c|}
\hline$\%$ & Average usage by hours \\
\hline$\% 6$ & Less than 2 hours \\
\hline$\% 76$ & 2 to 4 hours \\
\hline$\% 18$ & More than four hours \\
\hline
\end{tabular}

Figure 6 shows that $6 \%$ of respondents use social networking on less than two hours. $76 \%$ of the sample uses two to four hours a day, $18 \%$ use it more than four hours a day. The researcher explains that: The use of WhatsApp on social networks is very important in being a means of communication between girls have the advantages that it is easy and fast and achieve a virtual gathering environment for dialogue and chat of all kinds.

The researcher also refers to the Saudi girl's constant desire to keep pace with what is new in the world of technology, especially the social networks. Facebook used its first appearance at a high rate followed by its transformation to Twitter, the girl is easy and effective in communicating with others.

\section{Answer the fourth question What are the drivers of using WhatsApp?}




\section{Chart No. 8}

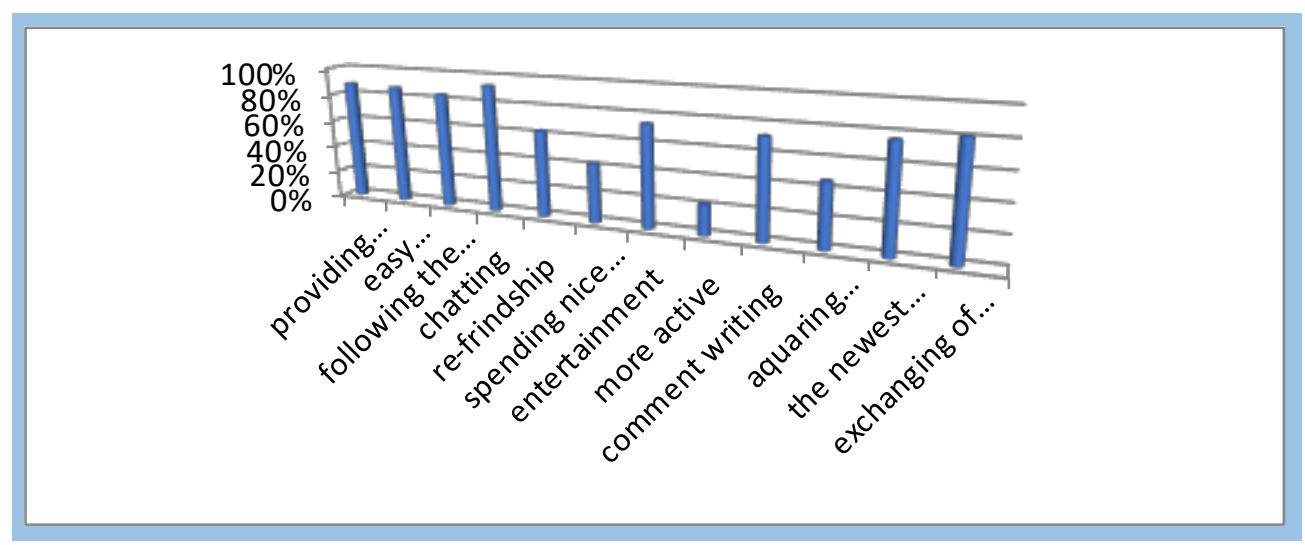

It is clear from chart No. 8 that the phrase "achieves the ability to chat and communicate instantly between a large number of friends at once" was $93 \%$, followed by "providing us with breaking news and quick information" with $88 \%$, followed by " Social, social and social "in general and on a large scale" by $87 \%$, followed by the phrase "a wonderful and distinctive and easy to use in the completion of the communication process" by $86 \%$ followed by the words "keep up with everything new and modern in technology", Followed by" Communication with Friends in the form of Groups and Exchange of Experience "by 81\%, followed by" Communication with Groups of Common Interest "at 76\%, followed by" Entertainment and Leisure by Habit or Habit "by 74\% The words "send messages to friends and associates and comment on their messages" by $72 \%$, followed by "restoring old friendships and easily helped the other party" by $63 \%$, followed by "deepening the world and gaining new skills and experiences" by $46 \%$, Followed by "spend time and get rid of the unit" by $43 \%$, followed by "to help me Activity and vitality and excitement "by $22 \%$.

The researcher explains that WhatsApp achieves the ability to chat and communicate instantly between a large number of friends at the same time "by creating groups of large numbers of friends who communicate with each other at the same time, with the touch of a button that connects the message to everyone at the same time.

\section{B- What are the disadvantages of the WhatsApp?}

\section{Chart No. 9}

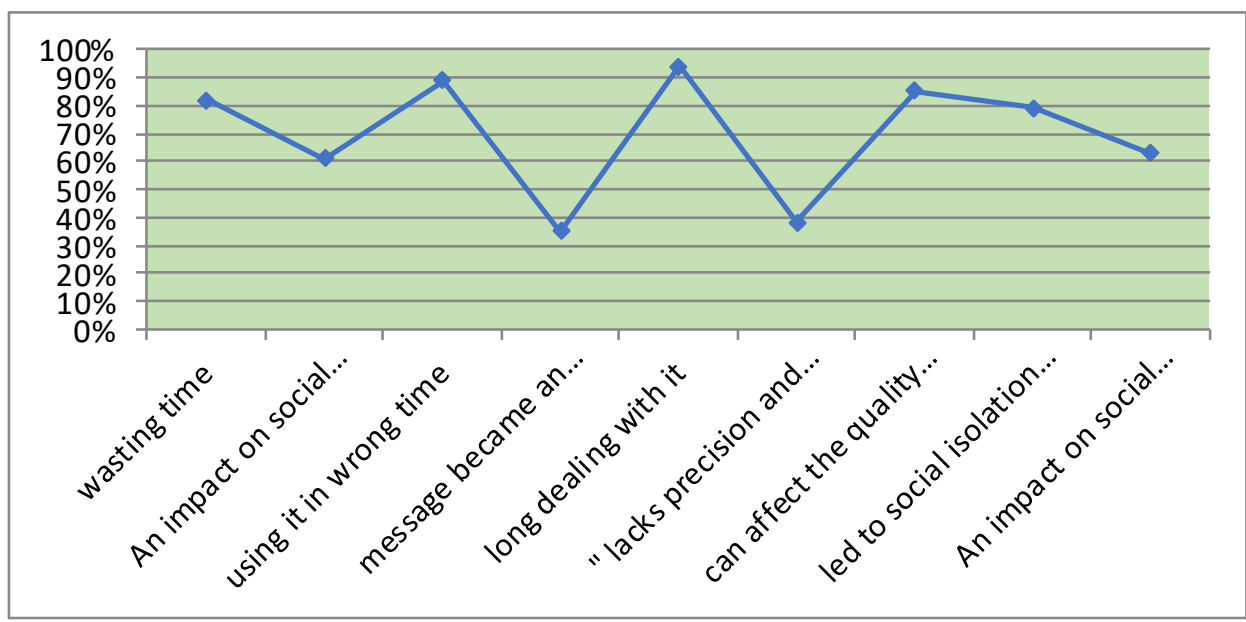

It is clear from chart No. 9 that the most important disadvantages of WhatsApp are: "Some people are totally addicted to the WhatsApp and are unable to dispense with 
them for even one minute and as they say they are going to get it," it was $94 \%$, followed by " Which can affect the quality of work during lecture times, which affects the focus of the lecture "by $89 \%$, followed by the words" students were busy listening to the teachers, noting during the lectures that students are busy communicating with others "by $85 \%$, followed by the words" One of the means of wasted time in the useless and frequent preoccupation with the priorities such as studying and focusing on lectures and family life by $82 \%$.

"An impact on social communication and his messages became an alternative to many visits that were of importance to the individual by" by $79 \%$, followed by the words "the proportion of historical statements false to prominent figures and the proportion of words and deeds to non-people and the development of prophetic traditions that have not been proven, call for creative things In the religion and the sayings of the nation's scholars who have influence on society, followed by the words "impact on the skills and arts of dealing with others and make us non-social, which led to social isolation and convergence," by $63 \%$, followed by "in family gatherings and gatherings of friends, they are engaged in talks on the WhatsApp that they can talk directly with these "61\%, followed by the phrase" lacks precision and credibility, most often the result of excessive dissemination of false news and rumors "by 38\%, followed by the words" Sometimes these massages are unsuitable for some persons.

The researcher explains that: Some people become completely addicted to the WhatsApp and cannot be dispensed with even for one minute and as they say they become immersed in it because the communication on the WhatsApp achieve the girls the virtual assembly of a large number of friends, which may be achieved in reality is difficult, They will use it to communicate and chat on topics they want.

The results of this study are in line with the results of the (sparklete, 2013) study, which concluded that the most important benefits of using the WhatsApp are the ability to send free messages to any international location free of charge, easy to use, import the phone calls automatically, no ads on the display, Lets you share your site with your friends.

The 2013 study added three advantages to using WhatsApp in sending messages and sending text messages without any money at all, the main feature of WhatsApp, because text messaging is very expensive, and teens like chatting and text messaging with this program Everything is free, so you can send an international message to one of your relatives or friends for free without worrying about the high phone bill. (duplicated)

This allows you to transfer all contacts from your address book, without transferring your contacts, The same results supported the results of the (cooldude, 2013) study, which found that the most important benefits of using WhatsApp are that it is one of the most prominent technological advances. People are increasingly using this technology because this technology has developed and facilitated many communications systems. The ease is provided that the other person is Contact him to be connected to the Internet, It works in one of the following operating systems: Windows, Blackberry, S, Android or Symbian, through which documents, pictures and songs can be exchanged through WhatsApp.

It also agreed with the results of the (SAR, 2012)study, which found that WhatsApp is the most important instant messaging application, enabling users to talk to friends, and the user base for Wizards is characterized by rapid and growing growth. It has already been proven that those who use the " Daily in millions of chatting on the Internet is fun only on the condition that the number of friends is large, which will actually be achieved because the WhatsApp program through the application of chat is becoming more popular in the world, dealing with billions of messages on a daily basis and the 
most important benefits Watts Group Chat Group: Chats Group is one of the most popular features on the watts app, where users can talk to up to ten of their friends at once, the chat feature is popular and unique, send attachments: you can certainly send pictures and video through Images and video message through $n$ Yeh ordinary but on WhatsApp, You can avoid the high costs of sending these files via SMS on the watt, and allow users to send voice recordings and other files to multiple destinations.

\section{The answer to the sixth question: How good is the WhatsApp program?}

\section{Chart 10}

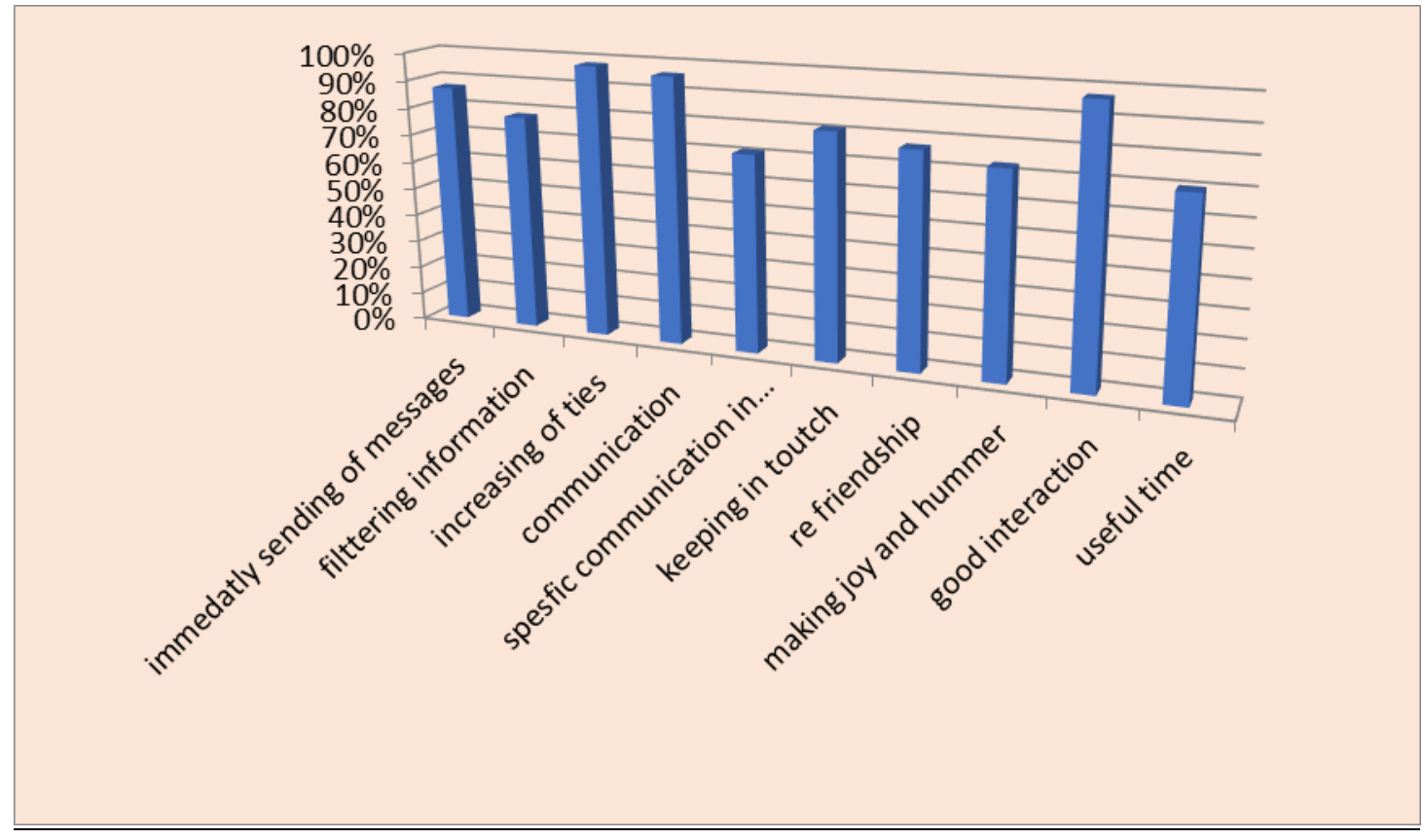

It is clear from chart 10 that the most important benefits from the use of WhatsApp are the ability of Group to communicate with family members, exchange family interests and increase social ties by $98 \%$, followed by an interaction between all community groups, especially in the exchange of congratulations on marriage, success and promotion. By $97 \%$, followed by an important phrase to connect with our friends at home abroad instead of the cost of regular messages and exchange pictures, video and voice calls by $96 \%$, It allows us to pay for the normal text messaging, providing a good space for interactivity and chatting, with $87 \%$ followed by "coverage of events, the development of instant news and major news feeds, and the speed of news transmission. In the media a few days later "by $81 \%$, allowing individuals to select the right information and broadcast it to others, diffuses the various artistic, academic and religious cultures and increases our cultural information" by $78 \%$, followed by the phrase "restoring old friendships, And his conversation with costs Less than $77 \%$, followed by "used as chat, entertainment and leisure time" by $76 \%$

"Seventy-seven percent of people were abducted from television, as a result of the fact that the correspondence made them a social, cultural, news and mouth-watering atmosphere", with $73 \%$, followed by "communication between peers or peers in the same field, and sharing and enriching experiences".

Table 7

\begin{tabular}{|c|c|}
\hline$\%$ & The Wats app Encryption program \\
\hline$\% 11$ & Yes \\
\hline
\end{tabular}




\section{$\% 89$}

No

The table shows that $89 \%$ of the study sample does not prefer to encrypt WhatsApp messages as opposed to $11 \%$ prefer. The results of this study differed from those of the study (Fernández, 2013)which concluded that encryption in the applications of instant messaging on social networks protects the privacy of the user during the conversations and maintains the privacy and security of the user.

The results of this study also differed with the results of the (Steven, 2012) study, which concluded that social networks have changed the way we communicate with people via instant messaging. We have completely switched from text messaging to instant messaging via Face book Tweets and WhatsApp, but certainly the WhatsApp messages are the most widely used, and since WhatsApp is the most widely used, messages must be encrypted or placed under internal control - there will definitely be a big difference if that happens.

The researcher explains that: The university girl uses the WhatsApp for chatting and communicating with friends, follow up the study news with her colleagues, and that the topics and conversations that they deal with do not have privacy and confidentiality, which requires encryption. But why is it important to encrypt the message? Because messages sent by users via the WhatsApp are very clear and have no encryption, so any other party, if it has a Wi-Fi connection, can access the messages in the network, which makes sense, especially for companies that exchange information Sensitive, there are many users who use personal or professional WhatsApp they can also capture information and share sensitive information via corporate Wi-Fi or what is much worse, than opening Wi-Fi in a public place like a mall.

B) What is the effect of the WhatsApp program on the values of the university girl?

Table (8)

\begin{tabular}{|c|c|}
\hline$\%$ & Effect of WhatsApp on the values of female university students \\
\hline$\% 22$ & Giving up some values such as honesty and honesty \\
\hline$\% 72$ & The spread of jokes and non-useful topics that bring abuse to others \\
\hline$\% 83$ & Not investing leisure time in useful work \\
\hline$\% 89$ & Lack of respect for the value of time and conversation of others \\
\hline
\end{tabular}

It is clear from the table that the value of "non-respect of the value of time and conversation of others at inappropriate times" received $89 \%$, followed by the value of "not investing leisure time in useful work" by $83 \%$ followed by the value of "the spread of jokes and useless topics that bring abuse to others" $72 \%$ followed by the value of "abandonment of some values such as honesty and honesty" by $22 \%$.

The researcher explains this: Because the entire WhatsApp service does not require any fees for this service, it is only enough to connect the phone to the Internet to enjoy this service even if the person uses it 24 hours a day. Continuous even at inappropriate times.

Study 103 recommended its theme: Parents Guides to Using Social Media "WhatsApp" It is always necessary to make sure to talk to your child about what is acceptable and what is inappropriate for sending and receiving messages.

Because the WhatsApp allows young people and teenagers to send text messages, videos, pictures and audio messages to one or more people with no message limits, once you install the application, you have your address book to see if anyone else you know already uses the WhatsApp and is automatically linked, There are ways to send anonymous messages. 
C) What are the advantages of messages in the wattage program?

Table No. 9

\begin{tabular}{|c|l|}
\hline$\%$ & \multicolumn{1}{|c|}{ The advantages of messages in the wattage program } \\
\hline$\% 100$ & Free messages \\
\hline$\% 92$ & New and persuading \\
\hline$\% 97$ & Voiceless receiving messages \\
\hline$\% 91$ & The exchange of files and transfer and comment and make full use of them \\
\hline$\% 100$ & Messages are not specified by words or time space \\
\hline$\% 98$ & Deals with billions of messages daily \\
\hline$\% 99$ & Voice and video messages \\
\hline
\end{tabular}

It is clear from the table that the words "messages are not expensive, they are free" and "messages are not defined by words or time zones" have been $100 \%$ higher, followed by "99\%," followed by "Handling billions of messages On a daily basis "by 98\%, followed by" $97 \%$ without receiving a voice alert "followed by" new and compelling message service "at $92 \%$

The researcher explained that: This service through modern social networking is the latest in modern science in the technology of communication, working through the Internet and thus his messages at no cost, enough to connect the phone to the Internet to enjoy this service, and the free of these messages are the reason for the huge increase in the number of messages and access to billions throughout the day, as it is not limited to simply communicate with the written words, but communicate with these messages in video and image or all of them all.

The results of this study were in line with the results of the (Arthur, 2012) study, which concluded that text was costing up to billions, and the telecom companies had already suffered huge losses resulting from the free messages that WhatsApp supports. This popular application allows users to use chat for free to solve place of contact.

I think we are facing a period of accelerated SMS erosion and this will not be a linear process on the WhatsApp, and it has grown tens of times a year, although the WhatsApp are a new phenomenon but they have already played a major role in a $25 \%$

"We have done this service and we are confident that it will provide a new and compelling experience and the number of users is unlimited and we have the capacity to afford all of our costs,"says Rahul Kumar.

\section{What are the advantages of instant messaging "conversations" on the wattage program?}

Table 10

\begin{tabular}{|c|l|}
\hline$\%$ & Chat or instant messaging features "Conversations" on the Watts program \\
\hline$\% 85$ & Scholar and non-scholar can use it easy. \\
\hline$\% 78$ & Young and old people can use it easy. \\
\hline$\% 99$ & Writing communication and audio and video messages \\
\hline$\% 98$ & Providing mini language to ease the using \\
\hline$\% 100$ & $\begin{array}{l}\text { This application is free of charge. It only requires the user to have Internet } \\
\text { access on its mobile }\end{array}$ \\
\hline$\% 97$ & $\begin{array}{l}\text { Represents a quantum leap and a vital breakthrough that has fundamentally } \\
\text { changed the means of communication in general and mobile in particular }\end{array}$ \\
\hline
\end{tabular}

The most important features of chat or instant messaging are the "free" conversations on this application. It only requires the user to have Internet access on its mobile. This is $100 \%$, followed by the words "users did not engage in a particular form of 
communication. Provide the possibility of communication in writing, audio or image "by $99 \%$, followed by the words" the provision of several languages made him nonmonopoly on the people of one language than the other but the same person can be used in more than one language depending on the language of the other party "by $98 \%$, followed by the words" Represents a quantum leap and a vital breakthrough that has fundamentally changed the means of communication in general and the transmission of doubt A special 97\% ", followed by the phrase" has become more accessible use "by $87 \%$, followed by the phrase" has become more popular science and became a nonscholar used "by $85 \%$.

The application is understood as "smart phone", which is called the smart phone revolution, and the WhatsApp has entered the market with great power and abundance. The WhatsApp is still the king of instant messaging, and the uniqueness of the WhatsApp is seen through Android and Apple devices. Singularity is free to use, not limited to writing but rather to sound and image, as well as not limited to one language but use it for several languages.

The results of this study are in line with the results of the (Hedlund, 2013)study, which concluded that WhatsApp is an easy-to-use application with many benefits such as: Lower costs: reduce your monthly bill for your mobile phone - rich messaging: Text is the most common way to communicate, in addition to including photos, videos, audio files and message icons, confirming a message that has been sent and received, group chats are easier on iTunes, where you can create a number of different groups with people.

The results of this study also agreed with the results of the (Sage, 2013) study, which found that the standard method of chatting has become an obsession for many. On the one hand, in terms of creation, you can create a special account on your personal phone. In terms of communication management, you should add a contact to someone's phone number. This method can be convenient. In terms of chat text, the core of all experiences is text messaging, exchange Files: BBM lets you share photos and videos only, while WhatsApp allows sharing photos and transferring other files and commenting on them.

In terms of chat group, both BBM and WhatsApp allow chat group and allow the exchange of images and video, and in terms of voice and video chat, both allow voice and video chat. In the dream of symbols and personalization both use codes and personalization, but the WhatsApp outstrip the BBM, Both status and profile support both the status structure and the profile.

Second: Indicative Statistics:

The statistical analysis is based on the idea of selecting a part of the "sample" society that is selected in a sound scientific manner, with the aim of using the data of this sample to arrive at possible results. To be distributed to the study community, and the most important means of testing hypotheses.

Interpretation of the first hypothesis:

There were statistically significant differences between the average scores of students at theoretical colleges and practical colleges in the use of watts app.

Table (11) Results of T-test (T) test to test the differences between theoretical and practical students.

\begin{tabular}{|c|c|c|c|c|}
\hline significance & T value & $\begin{array}{c}\text { Standard } \\
\text { Deviation }\end{array}$ & average & groups \\
\hline Sig. & 0.017 & 0.90 & 1.60 & Theoretical \\
\hline
\end{tabular}




\begin{tabular}{|l|l|l|l|l|}
\hline & & & & Colleges \\
\cline { 3 - 5 } & & 0.81 & 1.61 & $\begin{array}{l}\text { practical } \\
\text { colleges }\end{array}$ \\
\hline
\end{tabular}

The table shows that the value of $(\mathrm{T})$ between the average scores of students of theoretical colleges and practical colleges in the use of WhatsApp on social networking sites is not statistically significant.

This study, along with the results of the study agency: (Web, 2013), which found that from the applications of the new chat, our chat appeared new applications and fruitful and raised on the throne, such as WhatsApp, everyone has used it. The wattage now has more than 250 million monthly users on the site, a higher number than Twitter and Skype, originally called we chat, which is free text messages, voice messages and video calls, and was used by more than 400 million users already.

Interpretation of the second hypothesis: There are statistically significant differences between the levels of social communication (e.g., use / medium use / low use) and the use of WhatsApp.

Table (12) Test results: "One Way Analysis" (One Way ANOVAs) to compare responses of study subjects according to intensity of use.

\begin{tabular}{|c|c|c|c|c|c|}
\hline Sig. & T value & $\begin{array}{c}\text { Squares } \\
\text { average }\end{array}$ & $\begin{array}{c}\text { Free } \\
\text { space }\end{array}$ & $\begin{array}{c}\text { Total } \\
\text { squares }\end{array}$ & $\begin{array}{c}\text { Source of } \\
\text { variance }\end{array}$ \\
\hline \multirow{3}{*}{0.000} & \multirow{3}{*}{13.286} & 6.014 & 2 & 12.027 & among groups \\
\cline { 3 - 6 } & & 0.453 & 198 & 66.088 & In groups \\
\cline { 3 - 6 } & & 200 & 79.015 & total \\
\hline
\end{tabular}

It is clear from the table that the value of (q) for the intensity levels of social networks use is statistically significant, for the benefit of the extensive use of social networks, i.e., the use of social networks is more frequent for WhatsApp. This is a logical result, because the more people use the social networks, the more likely they are to use VoIP. The researcher explains that: social networks in general achieve a good virtual environment cannot be dispensed with, in addition to WhatsApp unique features and features not found in social networks, including ease of use and cheap cost of this communication medium, and can communicate with voice and all the picture and not words Only, and not specified by the number of words or characters such as Twitter "140", and not limited to one language, but uses multiple languages.

The results of this study were in line with the results of the (King, 2013)study. This study turned the answer to a central question: Why should we use the WhatsApp? The study answers that it works on all types of mobile phones. WhatsApp service is free, and when you install the program you must put your profile picture. Once you download the program, your profile picture will appear, and it is easy to get many numbers and connect with thousands of people.

Interpretation of the third hypothesis: There is a statistically significant relationship between the utilitarian motives of university girls' use of WhatsApp and the patterns of activity supported by the communication test the validity of this hypothesis, Pearson correlation coefficient was used to study the intensity of the correlation between two variables, the first in the utilitarian motives of the sample, and the second the types of activity supported by the communication effects of the sample when using the watts app. 
Int. J. Media. Mas. Com. Vol .2, No .07, 136-153 (2020)/

Table (13): Relationship of utilitarian motives with supported activity pattern effects

\begin{tabular}{|c|c|c|c|}
\hline significance & $\begin{array}{c}\text { significance } \\
\text { level }\end{array}$ & Pearson correlation & $\begin{array}{c}\text { Relationship of utilitarian } \\
\text { motives }\end{array}$ \\
\hline Sig. & 0.05 & 0.533 & $\begin{array}{c}\text { Relationship of utilitarian } \\
\text { motives for practical } \\
\text { students }\end{array}$ \\
\hline Sig. & 0.02 & 0.812 & $\begin{array}{c}\text { Motivational motivation of } \\
\text { theoretical students }\end{array}$ \\
\hline
\end{tabular}

It is clear from the previous table that the coefficient of correlation between the motivational motives of the practical students and the supported modes of activity is 0.532 , which is statistically significant at a significance level less than 0.05 . The correlation coefficient between the motivational motives of the theoretical students and the supported activity patterns is 0.812 , which is statistically significant at a lower level from 0.05

The results of this study are consistent with the results of the study of (Bakir, 2008), which found that there is a statistically significant relationship between the motivational motives of the parents and the supported activity patterns, and also the results of Pearson \& Rubin (1988) The researcher believes that the role of the family in supervising children should be activated in cases of owning mobile phones, especially school students, and directing them to the correct destination while consuming and receiving the products produced by these means. Not to give absolute confidence to the young man or girl, especially in the age group youth, which may be used by some of them negatively, but it is necessary to set limits, there is no harm.

Interpretation of the fourth hypothesis: There is a statistically significant relationship between the ritual motives of the university girl's use of the WhatsApp and the patterns of activity that are hindered by the communicative influences they exert at that use. To test the validity of this hypothesis, the Pearson correlation coefficient was used to study the intensity of the correlation between two variables, the first in the ritual motifs of the sample, and the second the patterns of activity with the communication effects of the sample when using the watt.

Table 14: Relation of utilitarian motives to supported activity patterns

\begin{tabular}{|c|c|c|c|}
\hline Sig. & $\begin{array}{c}\text { significance } \\
\text { level }\end{array}$ & Pearson correlation & $\begin{array}{c}\text { Rationale and Disability } \\
\text { Activity }\end{array}$ \\
\hline Sig. & 0.02 & 0.226 & Rationale of practical students \\
\hline Sig. & 0.05 & 0.565 & $\begin{array}{c}\text { The theoretical motives of } \\
\text { theoretical students }\end{array}$ \\
\hline
\end{tabular}

It is clear from the previous table that the coefficient of correlation between the ritual motives of the practical students and the types of disabled activity is 0,226 , which is statistically significant at a level less than 0.05 . This result is consistent with the outcome of the study of (Bakir, 2008), which found that there is a statistically significant relationship between the ritual motives of the parents and the patterns of disabled activity as well as the existence of a statistically significant relationship between the ritual motives of the mothers and the patterns of disabled activity (Rubin, 1997), which proved that ritualistic motives are the most active for the types of activity 
that are disabled to the effects at exposure. They are often less attentive to the series and more content, and more likely to avoid and divert attention to find the first alternative and be less affected by dramatic content.

\section{References}

Al-Mansour, M. (2012). The Impact of Social Media Networks on the Audience of a Recipient: A Comparative Study of Social Websites and Websites. Master Thesis in Media and Communication. Denmark: College of Arts and Education, Arab Academy in Denmark, 27.

Al-Qaoud, M. (2006). Designing a website to learn the Arabic Language for third grade primary students and measuring its effectiveness in learning to read and write . unpublished master thesis. Irbid- jordan : yarmouk university.

Arthur, C. (2012). Whats App: the new text messaging.

Bakir, M. A. (2008). Lifestyles that are reflected in the dubbed series presented in satellite TV and their suitability for the arab family. a survey study jounarl of media research No.30.

Chirinda, J. (2012). Face book, WhatsApp \& the transition of social networking to mobile advertisement. Retrieved from http://www.techzim.co.zw/2012/05/facebookwhatsapp-the-transition-of-social-networking-to-mobile/

cooldude. (2013).

Fernández, C. (2013). Completed multi-platform app that includes social network and instant messaging in the same tool Tuenti Social Messenger, the youth alternative to Whats app that is sweeping Spain, now available internationally for all platforms.

Goff, k. G. (2009). Child Safety and Technology on the Internet.

Hedlund, U. (2013). What's WhatsApp and how can I use it?

Kateb, S. (2012).

Khidr, N. Z. (2009, February 15-17). Psychological and social effects of arab youth using social networking sites. A case study on facebook users (cairo university) faculty of information media and family conference and the challenges of the times. Cairo University .

King, R. (2013). WhatsApp the Mobile Phone App that every PUA should use. Retrieved from http://puamethod.com/wordpress/whatsapp-the-mobile-phone-app-that-every-puashould-use/

Lund, E. (2013). High-quality, cross-platform messenger service "9.0 Awesome. Retrieved from http://whatsapp.appszoom.com/android

Murphy, P. (2012). why teens are not liking facebook but loving whatsapp. Retrieved from http://phil-murphy.com/why-teens-are-not-liking-facebook-but-loving-whatsapp/

Rubin, K. \&. (1997, April ). KIm, Jungle Rubin, M. Alan "The Variable Influence Of Audience Activity On Media Effects Unction Research Vol24 N02 .pp. P107 - 135.

Sacide Guzin Mezman, Y. K. (2009). The usage of social networks in educational context, International journal of human and social sciences.

Sage, S. (2013). cross-platform WhatsApp vs. BBM -messaging head-to-head.

SAR. (2012). Everything You Need to Know About WhatsApp.

sparklete. (2013). Disadvantage \& advantages of using whatsapp for pc. Retrieved from http://sparkletechnology.blogspot.com/2013/09/disadvantage-advantages-ofusing.html

Steven, C. (2012). The importance of encrypting messages in Whats App. Retrieved from http://techcrash.net/the-importance-of-encrypting-messages-in whatsapp /

Suzant.Kinney, A. R. (2012). Testing media richness theory in the new media: The effects of cues, feed back and task equivocality in forms on line HR.

Uwem Akpan, E. A. (2013). Uses and gratifications of social networking websites amoung 
youths . Nigeria : Department of communication Arts University of Uyo.

Web, D. (2013). The alternatives: Should Whats App really be scared"? Tasneem Akolawala. Retrieved from http://www.dnaindia.com/scitech/report-the-alternatives-shouldwhatsapp-really-be-scared-1866414. 